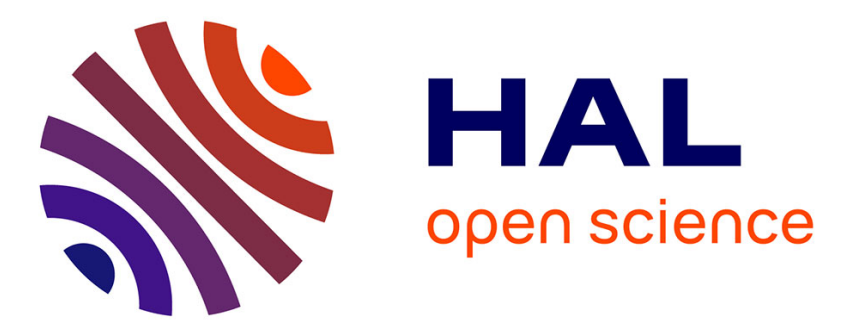

\title{
Quelle place prend la durabilité du bâtiment dans le processus de définition de la valeur du bien dans le contexte du marché français?
}

\author{
Frédéric Distler, Julien Husson, Fana Rasolofo-Distler
}

\section{To cite this version:}

Frédéric Distler, Julien Husson, Fana Rasolofo-Distler. Quelle place prend la durabilité du bâtiment dans le processus de définition de la valeur du bien dans le contexte du marché français?. Revue de l'Organisation Responsable, 2020, 15 (1), pp.55-65. 10.3917/ror.151.0055 . hal-02989747

\section{HAL Id: hal-02989747 \\ https://hal.science/hal-02989747}

Submitted on 5 Nov 2020

HAL is a multi-disciplinary open access archive for the deposit and dissemination of scientific research documents, whether they are published or not. The documents may come from teaching and research institutions in France or abroad, or from public or private research centers.
L'archive ouverte pluridisciplinaire $\mathbf{H A L}$, est destinée au dépôt et à la diffusion de documents scientifiques de niveau recherche, publiés ou non, émanant des établissements d'enseignement et de recherche français ou étrangers, des laboratoires publics ou privés. 


\title{
Quelle place prend la durabilité du bâtiment dans le processus de définition de la valeur du bien dans le contexte du marché français?
}

\author{
Frédéric Distler* \\ Maître de conférences associé, IAE de Metz \\ Frederic.distler@univ-lorraine.fr
}

\section{Julien Husson*}

Maître de conférences HDR, IAE de Metz

\section{Fana Rasolofo-Distler*}

Maître de conférences, IAE de Metz
*Université de Lorraine, IAE Metz - CEREFIGE, 1 rue Augustin Fresnel - BP 15100 - 57073 Metz Cedex 03

\section{Résumé}

Notre projet dans le cadre de cette recherche est d'observer au cours du processus de négociation/évaluation du bien la place qu'occupe pour les négociateurs la question de la durabilité. La durabilité du bien est-elle une tactique et un argument de vente à l'œuvre dans la construction des accords/désaccords dans la négociation autour du prix bien entre un professionnel (agent immobilier, marchand de bien, gestionnaire locatif, commercial, négociateur, promoteur...) et un « client » potentiel ? Nous allons ainsi, à partir de l'analyse de deux cas, tenter de comprendre l'enjeu de la durabilité du bâtiment dans le processus de fixation du prix et de la vente. 


\begin{abstract}
Our project as part of this research is to observe in the process of negotiation/evaluation of the property the place that the negotiators occupy the issue of sustainability. Is the sustainability of the property a tactic and a selling point at work in the construction of agreements/disagreements in the negotiation around the price well between a professional (Realtor, property dealer, rental Manager, commercial, negotiator, promoter...) and a potential "customer"? We will thus, from the analysis of two cases, try to understand the stakes of the sustainability of the building in the process of fixing the price and the sale.
\end{abstract}

\title{
Introduction
}

L'immobilier est un champ socio-économique fortement ancré dans l'économie de marché avec ses dérives, ses spéculations, ses opérations financières qui impacte l'environnement. Aussi, les pouvoirs publics, normes internationales, européennes, françaises, ont réglementé le secteur de la construction/vente à travers le constat de l'impact de l'immobilier sur l'environnement. Cette prise de conscience s'est matérialisée plus globalement par la signature du protocole de Kyoto en 2005 sur la réduction des émissions de gaz à effet de serre et le grenelle de l'environnement de 2007. Ces conventions vont ainsi mettre en mouvement le rapport Brundtland (1987) «notre avenir à tous » rédigé par la commission mondiale sur l'environnement et le développement de l'organisation des nations unies et présidé par Gro Harlem Brundtland. Ce rapport sera le paradigme et le point de départ d'une prise de conscience écologique et de l'impact de l'économie sur le bien-être des générations actuelles et futures. Boisnier (2015)

La transaction de biens immobilier se joue ici entre deux parties, le professionnel, détenteur de savoirs techniques et le particulier, détenteurs de savoirs empiriques acquis par recherche personnelle sur les sites spécialisés, journaux, rencontres... (Larceneux \& Parent, 2010) mais il peut aussi se jouer entre professionnels proprement dit et/ou entre particulier exclusivement.

Car en effet, selon (Larceneux \& Parent, 2010 P.P 166-167) «la fixation du prix de vente et l'évaluation du bien est un moment clé de la prise de mandat. C'est souvent à ce moment-là que le propriétaire retient, ou non, la proposition de l'agent. Or les intérêts du propriétaire et ceux de l'agent sont souvent divergents. L'agent immobilier souhaite un prix juste en dessous du prix du marché, afin de vendre vite et facilement, le propriétaire, quant à lui, veut vendre au plus chère, quitte à y mettre du temps».

Nous étudions dans le cadre de cette recherche la construction d'une négociation/évaluation entre un professionnel de l'immobilier, négociateur commercial, indépendant, qui est mandaté par un particulier pour acheter le bien, et un particulier qui vend son bien. Ces transactions s'inscrivent dans un processus de négociation dans lequel les divergences et les accords se construisent entre les acteurs. Le marché de l'immobilier, en France notamment, est hautement concurrentiel. Les particuliers, depuis la démocratisation des nouvelles technologies de l'information et des communications dans les années quatre-vingt-dix ont occupé progressivement l'espace des professionnels à travers l'accès à l'information et donc à l'expertise requise en matière de vente ou location de bien. Cette démocratisation d'accès à l'information a ainsi produit une concurrence en terme de connaissance de la valeur des biens, du marché, des techniques de construction, des modes de négociation, des taux d'emprunt... entre les professionnels de l'immobilier, notamment les agents immobiliers et les particuliers 
attachés à la location ou à la vente de leur bien, Rivaton, Pavanello (2017). Aussi, cet état de fait nous conduit à définir la problématique suivante : quelle place prend la question de la durabilité dans le processus de transaction, évaluation et définition du prix d'un bien immobilier?

Depuis le grenelle de l'environnement en 2007, la question de l'écologie des bâtiments est une préoccupation majeure des politiques publiques en matière de logements mais également un enjeu pour la rentabilité économique de l'habiter. En effet, les économies d'énergie impacts le niveau de vie des propriétaires et locataires des bâtiments à usage résidentiel, commerciales et industriels. Cet enjeu sur la qualité de vie et la préservation des ressources naturelles engage la responsabilité sociale des entreprises et des citoyens locataires et propriétaires, (Capron \& Quairel-Lanoizelée, 2007). Aussi, les transactions de biens immobiliers sont lestées par la question de l'éco-énergie du bâtiment comme marqueur de sa qualité et de sa rentabilité et donc de sa profitabilité.

\section{De la sociologie économique}

Les récents travaux de la sociologie économique, Callon, (2017), Vatin, 2009, Karpik, (2007), Cusin, (2004), Trigilia, (2002), Fligstein, (2001), Zelizer (2001), Granovetter, (2000), Callon, Méadel, Rabeharisoa, (2000), Steiner, (1998), Favereau (1989)... permettent d'appréhender les processus de définition-construction de la mesure, de l'évaluation et au final de l'attribution d'une qualificationdéfinition d'un bien et d'un prix. En partant des difficultés de coordination au sein des marchés caractérisés par des biens complexes, singuliers ou incommensurables, que l'économie néoclassique ignore, (Karpik 2007)' elle invite à considérer les opérations par lesquelles l'échange devient possible : il s'agit notamment de voir comment se met en forme un processus de négociation-transaction et plus largement, les dispositifs mobilisés (d'expertise, de calcul, de fidélisation, etc.). Les acteurs de l'échange parviennent à qualifier les biens mis en vente et à élaborer les jugements qui rendront possibles leurs choix, (Dubuisson-Quellier 2018) La sociologie des qualités de marché se fixe comme programme l'étude de la production et de l'activation de ces dispositifs qui, contrairement à la fiction du marché néoclassique où le seul dispositif de ce type est l'abstraction du commissaire-priseur, peuplent et structurent le marché (Cochoy Franck \& Dubuisson-Quellier, 2000)

\section{1 ...à la sociologie économique des qualités}

Ce schéma de compréhension est particulièrement pertinent pour l'achat et la vente de biens immobiliers : les caractéristiques et la qualité des logements ne sont pas facilement observables, l'information sur l'offre est incomplète, les mécanismes d'évaluation et de fixation des prix restent mal connus des particuliers et l'importance des comportements spéculatifs accroît encore l'incertitude sur les prix, surtout dans l'ancien. Par ailleurs, l'offre est dispersée, chaque logement pouvant être considéré comme unique, et il n'y a pas de lieu fixe où se rencontrent l'offre et la demande, pas de lieu de marché, ni de dispositif régulateur, comme un organisme de cotation ou un commissaire-priseur, (Colloos et al 1997). Contrairement à beaucoup de travaux en sociologie économique toutefois, notre point de départ n'est pas l'incertitude à laquelle sont confrontés les acheteurs et les vendeurs, mais bien l'activité et les pratiques des agents immobiliers dans le processus de vente et les réactions-ajustements des vendeurs/acheteurs. Ce point de vue permet de donner toute son importance à la pré construction des ajustements par la constitution d'un espace de négociation que (Callon 2017), nomme le «cadrage », c'est un aspect essentiel de l'intermédiation immobilière. Notre approche se caractérise également par une insistance sur les différences de représentation dans l'acte de transaction/négociation étudiées, ce qui permet de restituer le rôle des caractéristiques sociales des acteurs impliqués dans la vente. Car en effet, vendre un bien immobilier engage 
des acteurs et les conduits à mobiliser un certain nombre de ressources. Pour le professionnel de l'immobilier, négociateur indépendant ici mobilisé dans le cadre des deux situations, ces dernières sont des savoirs d'expertise, d'expérience mais aussi et surtout un savoir orienté vers une finalité qui est celle de vendre et/ou d'acheter un bien. Le vendeur, dans notre cas un particulier, mobilise des savoirs que nous qualifions ici de savoir « d'usage ». Ils sont fondés sur l'appropriation de connaissance acquise dans le cadre de recherches de biens sur de multiples supports tels que sites web, journaux, revues, interconnaissances.... Entre un professionnel et un vendeur dans le cas d'une transaction se joue ainsi un processus de négociation/évaluation du bien à partir d'une volonté commune, vendre et/ou acheter le bien. Cette transaction s'origine dans un processus métrologique. En effet, le bien en question n'a cessé d'être objet d'évaluation depuis sa conception jusqu'à sa vente actuelle. Aussi, le bien se trouve sur un marché, ce dernier est défini comme " un dispositif agonistique ou s'affrontent et $s$ 'éprouvent des définitions contradictoires des biens et de leur qualification. Ces affrontements se résolvent dans des compromis qui prennent la forme de transaction donnant lieu à des paiements monétaires. »(Callon, 2009.p. 249 ;). Mais comment sont constitués les valeurs monétaires ainsi définies sur le marché ? Pour (Callon. 2009. P.252), la notion de valuation est utile pour «désigner l'ensemble des récits, mécanismes, dispositifs, outils qui constituent les valeurs et, simultanément, mettent en place leur mesure. Cette définition conduit à envisager la forme particulière de valuation qu'est la valuation marchande, comme un processus qui qualifie des biens et qui, à chaque étape de ce processus, attribue à leurs qualités ainsi constituées une valeur monétaire rendant possible le calcul de prix relatif. "

\subsection{La « valuation » comme modalité d'évaluation/singularisation du bien}

La valuation est donc ici mobilisée comme étant un processus qui qualifie un bien et lui attribue une valeur. Dans nos cas présentés ci-dessous, les échanges entre les acteurs sont traversés par des modalités de valuation fondées sur la valeur, affective, espérée et négociée. La valeur du bien est ainsi une détermination de sa qualité et une définition de son prix. Le bien est singularisé, détaché de toute contingence, il est objet de transaction pour ce qu'il est. En effet, (Callon, 2017 P.320) indique que «dans la transaction bilatérale (un vendeur $V$, un acheteur $A$, et un bien enjeu de la transaction), l'ensemble des calculs possibles est effectué par les agents. Les compétences calculatrices requises sont élémentaires mais très strictes. Il faut et il suffit que V sache au-dessus de quel prix (pv) il acceptera de vendre et que A sache au-dessus de quel prix (pa) il refusera d'acheter. Il existe finalement une multitude de prix envisageables, compris entre pv et pa. Le prix finalement retenu dépend de la négociation (le marchandage) entre les deux agents et par conséquent des rapports de forces qui incluent notamment, mais pas uniquement, l'habileté rhétorique, le chantage, une meilleure information, les positions de pouvoir, la coutume, la manipulation des mécanismes cognitifs. La fixation du prix dans la transaction bilatérale résulte donc de la combinaison d'activités calculatoires et de rapports de forces $\gg$.

Ce sont ces rapports de force et ses combinaisons calculatoires qui sont ici objet de notre étude à travers la description et l'analyse de deux situations de vente de biens immobilier entre un professionnel (négociateur commercial en immobilier), et un particulier. Le contexte de la vente, les arguments déployés par les acteurs, les stratégies mises en œuvre autour de la durabilité comme vecteur favorisant une hausse ou baisse du prix, tous ces paramètres sont des éléments qui concourent à fixer le cadrage de la transaction.

Ainsi, notre analyse de deux situations de négociation et évaluation de bien ci-dessous décrites nous conduit à formuler la conjecture suivante : l'argument de durabilité est un cadre 
« stratégique » dans lequel vendeur et acheteur achoppent pour définir le prix. Le prix est ici un long processus de négociation qui s'inscrit dans un cadrage (Callon, 2017) qui mêle tactique, ruse, conviction, arguments rationnels, émotions, atermoiements et singularisation dans la vente et ou la location d'un bien. Le bien convoité peut toujours faire l'objet ultérieurement d'aménagement durable selon les possibles vendeurs ou acquéreurs. Le prix et l'emplacement ne sont pas les seuls critères qui emportent la décision sur fond de « coup de cœur », mais la définition habile de la singularité du bien et donc de ses particularités. La durabilité du bâtiment est définie par les acteurs au regard des économies d'énergie souhaitées (eau, électricité, chauffage...). C'est sur ce point économique que le vendeur axe notamment ses arguments mais pas seulement. Au regard de la spécificité du bien, donc de sa singularité, l'éco-énergie est soit un atout dans les arguments avancés pour le vendeur, (le bâtiment est peu énergivore), soit une « occasion » pour l'acquéreur, (le bâtiment est énergivore, il faut engager des travaux), donc baisser le prix de vente. Dans tous les cas, l'argument éco énergétique est un élément majeur dans la prise en compte de l'évaluation du bien et de son prix. La durabilité évoquée et mobilisée par les parties comme étant un élément sur lequel fonder la valeur du bien est le point de passage obligé dans la négociation. L'éco-énergie est traduite dans le discours des transactionnaires par un certain nombre d'éléments tels que ; un investissement sur le long terme; des économies substantielles ; une mise aux normes du bâtiment ; des conditions d'habitat plus saines... ces arguments sont étayés et légitimés par l'outil dit « de diagnostic de performance énergétique ». Cet outil sert à mesurer graduellement par code couleur les modalités de la consommation en énergie du bâtiment. Il est une référence "objective» dans la définition du profil éco énergétique du bâtiment. C'est un actant qui lie des arguments noués autour du calcul potentiel de travaux. Il agit sur la transaction par son caractère de neutralité, d'objectivité fondée sur la mesure chiffrée. Or, cet actant est un outil qui permet l'interprétation que les transactionnaires donnent aux chiffres, qui contribue à définir un espace d'échange dans lequel se joue la fabrication de la valuation du bien et la production de chiffres. Ces derniers disent les travaux à opérer, disent l'état du bien en général. Aussi, viennent corroborer, compléter, affiner, démentir, ou contester cette instance calculatoire qu'est le DPE, les factures, les travaux récents, les projets de réfection, les actes de copropriétés, des engagements à travaux... en fait la problématique de la durabilité des bâtiments dans le cadre d'une transaction est devenu un fait majeur qui contribue à orienter et structurer la négociation/évaluation du bien. Aussi, qu'appelle-t-on bâtiment durable?

\subsection{Le concept de bâtiment durable}

Selon Primault 2015 (P. 34), La durabilité d'un bâtiment se définit comme "un bâtiment performant du point de vue de l'énergie et de l'environnement mais aussi du point de vue des aspects sociaux et de sa durabilité sur l'exemple de son cycle de vie (de la construction à la démolition en passant par la phase d'exploitation du bâtiment proprement dite). Cette définition inclus des considérations sur le confort des occupants, l'accessibilité sociale, la mixité, la flexibilité des locaux et la résilience dans le temps de la construction. En effet, Paul (2011) souligne que Le secteur du bâtiment est un secteur stratégique pour le développement durable puisqu'il représente près de $43 \%$ de la consommation d'énergie finale française, $23 \%$ des émissions de gaz à effet de serre, $50 \%$ de la production de déchets.

Ducroux (2002), précise que les politiques se sont progressivement emparés de cet enjeu à travers des réglementations sans cesse plus coercitives alors que les certifications environnementales ont ouvert de nouvelles perspectives pour les propriétaires afin de 
différencier leurs biens. L'arrivée de bâtiments très performants d'un point de vue environnemental ainsi que l'objectif de la loi Grenelle 2 de réduction de $38 \%$ de la consommation d'énergie du parc de bâtiment a introduit une rupture entre d'une part des bâtiments neufs très performants et d'autre part un stock de bâtiments menacé par une obsolescence réglementaire. C'est un enjeu pour les promoteurs/constructeurs, car l'intégration des normes de durabilité dans la construction, le choix des matériaux, la recherche de foncier, l'impact des travaux sur l'environnement dessinent de nouveaux défis en termes de coûts et d'adaptation des bâtiments aux exigences d'une vie plus saine en harmonie avec l'environnement. Nappi-Choulet (2009).

Inscrit dans une politique globale de développement durable, le bâtiment du grenelle de l'environnement définit les normes vers lesquelles doivent tendre les constructeurs et les bailleurs pour atteindre une performance énergétique de nature à préserver l'écosystème, le cadre de vie, la santé, dans le but de sauvegarder notre bien commun qu'est la ressource naturelle. En effet comme indiqué dans le grenelle dit 1 « le secteur du bâtiment génère près du quart des émissions de gaz à effet de serre ». Aussi, l'enjeu du grenelle est d'obtenir une baisse de la consommation d'énergie des bâtiments. Amarillo (2013)

En matière de vente ou location d'un bien immobilier, l'outil emblématique qui caractérise cette politique est représenté par le DPE (diagnostic de performance énergétique). En effet, son objectif est de réduire les émissions de $\mathrm{CO}_{2}$ et contribuer aux économies d'énergies. Il a pour fonction de mesurer les consommations d'énergies et d'informer le locataire ou propriétaire de cette consommation. Aussi, cet outil, dans le cadre de l'évaluation et de la négociation d'un bien immobilier apparait être un outil stratégique qui permet d'énoncer, appuyer, commenter, interpréter, les données relatives à la consommation d'énergie du bâtiment et à sa participation à « l'effort » que requiert le développement durable auprès de tout citoyens/habitants.

Selon Primault 2015 (P.35), il existe de nombreux autres référentiels qui évaluent la performance environnementale et sociale des bâtiments - Des certifications et labels validés par des tiers : "certification HQE française, référentiel BREEAM anglais, certification CASBEE japonaise, certification DGNB... Ils sont basés sur l'évaluation d'un référentiel basés sur divers indicateurs physiques et des seuils à atteindre. Les résultats sont alors agrégés par cible puis globalement en fonction de pondérations. Des dispositifs de notations environnementales des bâtiments, en particulier Le "Green Rating》 Il s'agit d'un système de mesure de la performance environnementale des bâtiments existants établi par Bureau Veritas en collaboration avec AEW Europe, AXA REIM, GE Real Estate et ING REIM. Il propose une méthodologie d'évaluation en considérant les 6 thématiques suivantes : consommation énergétique, émissions CO2, eau, transports, déchets, santé/confort. Pour chacune de ces thématiques, 4 niveaux de performance sont évalués : la performance intrinsèque basée sur des usages conventionnels, la performance actuelle du bâtiment prenant en compte ses conditions d'utilisation en cours, la performance actuelle potentielle estimant sa performance en cas d'optimisation de l'exploitation et de l'usage, la performance intrinsèque potentielle estimant sa performance après rénovation pour un montant d'investissement normalisé ». Partant de cet outillage technique d'évaluation et de mesure de la performance d'un bâtiment durable, le DPE est celui imposé par la réglementation en vigueur en matière d'évaluation de la consommation d'énergie dans le cadre d'une cession, vente ou location d'un bien immobilier. Cet outil d'évaluation/repère pour les acteurs est un dispositif qui participe pleinement à la négociation pour la vente ou la location du bien. Aussi, selon Stimec (2011) cette rencontre se déroule dans un cadre, jamais définit à l'avance, au sein duquel se déroule le processus de négociation. Ce dernier est construit par les interactions des négociateurs qui contribuent à définir le cadrage 
dans lequel ils négocient la détermination du prix et ainsi la singularisation du bien objet de négociation/valuation.

\section{Le cadre conceptuel mobilisé : Singulariser le bien}

Nous inscrivons notre recherche dans le sillage conceptuel des travaux de Callon (2017) relatifs à l'économie des qualités et ceux de Vatin et al (2009), qui portent sur l'évaluation et la mesure des biens et services. Ces auteurs sont complémentaires car ils conçoivent les marchés, les biens et services comme étant des singularités portées par des qualités intrinsèques définies par les acteurs en amont et aval de la transaction. En effet, le bien, le service et le prix sont la résultante de processus de définition depuis la conception jusqu'à l'action de transaction marchande. La valeur (provisoire) d'un bien et/ou d'un service est l'issu d'un processus de création de valeur sur le marché. Cette valeur émane et s'éprouve, en amont du marché, "via les opérations pratiques par lesquelles les biens et les services sont mesurés, évalués, élaborés techniquement et économiquement par les organisations productives qui édictent des normes de gestion, mais aussi les agents qui ont la charge de les mettre en œuvre, et ne peuvent le faire qu'en les contournant, les reformulant, les combinant avec leurs propres axiologies. »Vatin,( P.27. 2009).

Callon (2017)... et Vatin (2009) s'inscrivent tous deux dans une sociologie économique préoccupée par la métrologisation du monde, c'est-à-dire par les multiples dispositifs techniques de mesures du monde. Callon et al (2000) rejette l'idée que l'offre et la demande seraient deux espaces homogènes qui se rencontreraient sur un marché et de leurs confrontations un prix serait fixé. Pour Callon (2017), quel que soit le produit, l'offre et la demande sont des « tourbillons ». Par là il faut comprendre que les clients n'existent pas a priori et que, pour vendre, il convient d'effectuer toute une série d'opérations et se munir de nombreux instruments. Selon Callon (2017), les marchés sont des agencements marchands, ils ne sont jamais abstraits. Ils se matérialisent toujours dans un décor matériel (une foire, une salle de marché...) dans lequel l'échange prend effectivement corps. Les agencements marchands, dont l'objectif est de promouvoir des transactions bilatérales, fonctionnent d'autant mieux qu'ils favorisent la singularité du produit vendu, ce qui nécessite donc de l'innovation et qu'ils permettent un ajustement continu entre offreurs et vendeurs (ce qui implique la mobilisation d'une foule de personnes, chercheurs, banquiers, organismes de certification, associations de consommateurs, etc.). Afin d'expliciter sa démonstration, Callon (2017) utilise de nombreux concepts originaux, dont celui de cadrage. Cette notion désigne les dispositifs orientant et structurant l'action des offreurs et des demandeurs. Le prix est ainsi le produit de cadrages, de mises en forme, de réglages, de négociations, sur lequel se mettent d'accord les vendeurs et les acheteurs. Aussi, Il est d'abord l'expression d'agencements marchands et donc le produit d'interactions sociales multiples et évolutives.

Il s'agit ainsi pour Callon (2017) et Vatin (2009) de mesurer la formation de la valeur économique dans ses dispositifs de mesure. Contrairement au paradigme de la théorie économique néo-classique dissociant l'évaluation de la valorisation, les auteurs entendent identifier les espaces métrologiques élaborés par les acteurs, le sens qu'ils leur donnent et l'effet de ces dispositifs de mesure sur les relations sociales. Entendons que la valeur économique d'un produit (ou d'un service) n'est pas réductible à sa valorisation sur la scène marchande : « Le processus (agonistique) de qualification-définition des biens est formaté par la perspective de l'échange et, réciproquement, l'échange n'est qu'un moment du processus, jamais achevé, de qualification et de requalification des biens, dont il ne peut être séparé » (Vatin, 2009. p. 251). Dans les cas étudiés, la valuation du bien s'est traduite par la mobilisation pour le professionnel 
d'un certain nombre de critères pour appuyer son expertise. Ces critères qui constituent le cadrage rhétorique de la situation sont le lieu et l'emplacement du bien, son prix, son état, ses caractéristiques, sa revente potentielle, sa rentabilité en terme de consommation d'énergie. Aussi la question de la durabilité du bien est posée en termes de rentabilité économique et sociale. Les préoccupations d'ordre écologique, sociétale et donc civique et éthiques sont peu ou pas abordées par les négociateurs. Le bien est d'abord un actif qu'il s'agit de rentabiliser financièrement d'une part et d'autre part d'y trouver un confort de vie qui sied à la définition que l'on se donne du bien vivre. Or, la notion de bien vivre est éminemment subjective car elle recouvre des valeurs et préférences propres à tout un chacun au regard de ses expériences et représentations qu'il se fait de son rapport à l'habitat. Larceneux \& Parent (2010

\subsection{Méthodologie mise en œuvre}

Nous inscrivons notre méthode de recherche dans ce que Callon (2012) nomme les dispositifs agonistiques, à savoir les marchés dans lesquels «s'affrontent et s'éprouvent des définitions contradictoires des biens et de leur qualification ». Ces affrontements se résolvent dans des compromis qui prennent la forme de transactions donnant lieu à des paiements monétaires »

L'objectif ici est d'analyser et expliquer les modalités du compromis à l'aune du concept de valuation.

Nous présentons deux cas de négociation dans le cadre de transaction d'une maison individuelle et d'un immeuble de trois étages. Les transactionnaires sont des professionnels qualifiés de négociateurs en immobilier indépendant et des particuliers qui souhaitent vendre leur bien. Nous percevons dans ces processus d'échange les rationalités à l'œuvre faites de construction d'arguments, de tactiques, de persuasion, d'émotions, en bref des formes d'actions qui s'éprouvent dans un contexte interrelationnel qui forme le cadre dans lequel se déroule la négociation relative à la transaction du bien. Cette négociation est objet d'évaluations diverses sur la nature du bien, ses caractéristiques techniques, son emplacement, son prix de marché, sa durabilité. La valeur du bien s'inscrit ainsi dans une configuration relationnelle dont la finalité est de mesurer et évaluer les arguments et les caractéristiques du bien. C'est un processus d'individualisation et de singularisation du bien qui est à l'œuvre dans la détermination du prix. Il s'agit de l'isoler des autres biens constitutifs de l'environnement (le marché), pour l'identifier et lui définir une valeur socio-économique.

\subsection{Notre positionnement méthodologique : l'étude de cas}

Nous faisons le choix de l'étude de cas comme processus d'analyse pour plusieurs raisons. Elle permet la mise en œuvre d'une stratégie de recherche qui polarise son attention sur l'analyse des processus de décision et sur l'élaboration de concepts opératoires. Cette technique d'analyse favorise la construction de modèle théorique à partir de la description et de la mise en perspective des dynamiques à l'œuvre dans l'organisation (Hlady-Rispal, 2000). Elle a comme visée méthodologique, de comprendre, par interprétation, les motivations et les raisons que se donnent les acteurs d'agir comme ils le font dans des contextes définis. Le positionnement adopté est ici clairement intuitiviste, à savoir, comprendre les motivations des acteurs et les resituer dans leur contexte d'énonciation temporel et significatif. Il s'agit d'adopter une attitude compréhensive et empathique afin d'appréhender le sens que les acteurs donnent à leur comportement (Weber, 1995). Nous avons croisé, dans le but d'optimiser la validité de notre recherche, plusieurs sources d'information (écrites et orales), différents modes d'enquête (entretiens et observations de situations). Les informations écrites émanent d'une revue de la 
littérature. Ce croisement des données articulé à l'analyse du corpus duquel a émané une trame conceptuelle nous conduit à « comprendre » les raisons et les multiples facettes du déploiement des arguments et autres tactiques dans le cadre de la négociation relative à la vente et ou à la location d'un bien immeuble.

\subsection{Le processus de valuation du bien : entre expertise et savoirs profanes}

Dubard, tripier, Boussard (2015) définissent la notion de savoirs experts comme étant fondé sur des savoirs techniques, économiques, relationnels, commerciaux, comptables, financiers détenues par les professionnels d'un secteur. Ces savoirs sont au fondement de leur légitimité professionnelle et de leur compétence. Ils contribuent à leur reconnaissance. Les savoirs profanes sont des connaissances et savoir-faire acquis par autoformation et expérience. Ils participent à l'orientation de l'action et des croyances des acteurs. Ils sont des représentations à priori des champs sociaux dans lesquels ils éprouvent leurs savoirs et savoir-faire, Bacqué, Biewener, (2015). Selon Trepos (1996), ces formes de savoirs (experts et profanes) concourent à la construction des catégories de pensée et de légitimité des discours concurrents dans le cadre d'échanges négociés. La présentation et l'analyse de deux cas ci-dessous nous apparaît être illustrative d'un processus de définition de la valeur à partir des mécanismes de métrologisation induite par les échanges qui forment la négociation/évaluation du bien. Le bien est localisé, défini, inscrit dans une histoire subjective et donc affective et redéfini à partir des modalités de calculs dont l'objectif est de construire un prix résultat d'un consensus autour de valeurs mobilisées dans le cadre de la transaction.

Cas $n^{\circ} 1$. Vendre la maison familiale : entre références techniques et affectives

Madame Martin vend sa maison dans laquelle elle a grandi et à vue grandir ses enfants. Veuve depuis plusieurs années, elle y vit seule. Trop grande pour elle, c'est avec regret dit-elle qu'elle a pris la décision de vendre pour s'installer chez sa fille ainée. En lien avec sa fille, son fils et son gendre, un prix a été fixé, « aux environs de 180000 euros dit-elle. La définition du prix n'a cessé d'évoluer au gré des recherches d'information sur les multiples sites immobiliers. Les comparaisons de prix, les échanges argumentés avec les membres de sa famille, ses amis (es), ses voisins, les doutes sur la valeur approximative de la maison ainsi définie, la crainte de ne pas être dans la bonne fourchette, de se faire «avoir» engendre chez Madame Martin une indétermination du prix et de la valeur globale du bien. Elle évoque le fait que les acheteurs se référaient au prix du marché sans tenir compte de tous les agréments et le potentiel que recèle la maison, selon Madame Martin «L'agent immobilier a évalué ma maison par rapport au prix du marché disait-il, il a compté les pièces, mesuré les espaces habitables, et a défini un prix fourchette, or les nombreux travaux de maintenance, de réfection, de construction, d'aménagement ne semblaient pas avoir d'intérêt pour lui. Elle indiquait que les candidatsacheteurs ne se préoccupaient que de la négociation du prix...à la baisse, " toujours trop bas ", en dessous de la valeur estimée par elle-même. La mise en avant de l'entretien de la maison pour qu'elle reste performante et « habitable » ne semblait pas être un sujet d'intérêt immédiat pour les acheteurs, était-ce une stratégie de leur part? Feignaient-ils de s'en désintéresser? Cette stratégie fondée sur de multiples tactiques comme la feinte, la dévaluation, le désintérêt, l'estimation de travaux à réaliser, le surcoût d'entrée et de mise aux normes...est souvent utilisée dans le cadre de vente d'un bien immobilier selon le négociateur commercial. Il explique que la définition du prix par les parties engendre une multitude de tactiques possibles pour évaluer soit à la hausse, soit à la baisse. En effet, selon l'agent immobilier « acheter ou 
vendre un bien implique de développer des compétences acquises comme savoir convaincre d'un prix, ignorer sciemment telles données, minimiser l'importance de telles autres, aussi il faut parfois savoir bluffer, c'est un jeu ou le plus fin tacticien remporte la mise».

La confrontation des rationalités, fondées sur le savoir expert pour le représentant de l'acheteur, (savoir technique, mesure du prix à partir du marché de référence, technique du bâtiment, estimation des travaux à réaliser...) et sur le savoir d'usage pour le vendeur, (surestimation du prix en décalage avec le prix du marché connu, méconnaissance du coût des travaux à réaliser, des économies d'énergie supputées, difficile interprétation des diagnostics...) dessine l'espace de négociation, de tension, d'argumentation, de stratégies, de rapports de force au sein de laquelle se définit un prix en comparaison des biens de même nature sur le marché local. Madame Martin, à la suite de moultes échanges avec divers agents immobiliers, finira par se rallier aux préconisations des professionnels qui adossent leurs arguments sur des critères techniques qui se veulent neutres, objectifs et par conséquent valables à ses yeux. Mais elle s'est décidée à la suite de visites de particuliers qui «tenaient des discours tendant à dire que le prix n'était pas ceux du marché au vu de la localisation du bien et de sa configuration et de son état général ». Mme Martin. La valeur qu'attribue Madame Martin, comme indiqué plus haut, à son bien est construit sur la représentation qu'elle se fait du prix de sa maison. Mme Martin a fait des démarches dans les agences immobilières locales, a consultés les sites spécialisés d'offres et de demandes de biens, a surfé sur des forums de vendeur de biens similaire au sien pour se faire une idée du prix et des conditions de ventes. Selon Mme Martin " j'ai une connaissance du marché du pavillon dans l'ancien. Ma maison compte quatre chambres, salon, salle à manger, une cave et grenier, un garage, et trois cent mètre carré de potager et verger. Il y a des réfections, notamment changer les fenêtres pour les mettre aux normes BBC (bâtiment basse consommation) et rafraichir les peintures et papiers peints ». Or, selon elle, le négociateur insistait sur la nécessité de changer la chaudière au gaz car trop ancienne, plus aux normes, trop énergivore, ainsi que les fenêtres et la porte de garage « trop vétuste $»$.

Selon le négociateur, négocier le prix d'un bien à la baisse passe par l'inventaire des réfections à engager. Elles sont d'autant plus entendues qu'elles induisent des économies d'énergies pour l'avenir. Les multiples dispositifs fiscaux incitatifs sont des leviers qui permettent de justifier la nécessité de se mettre aux normes de l'éco-énergie. Le vendeur, ou l'acheteur résiste peu à ses arguments. Soit il laisse tomber le bien, soit il achète et se lance dans un plan de financement pour mettre aux normes le bâtiment. Aussi, la rationalité technique/économique conduit à imposer ses normes dans l'échange, à contraindre le vendeur à revoir son estimation, à ignorer l'aspect affectif au bénéfice de l'aspect pécuniaire. Dans ce cadre l'aspect de durabilité est un élément qui vient renforcer la rationalité technique dans la détermination du prix au regard de l'emplacement et du marché. Dans l'hypothèse où les travaux d'éco-énergie serait à effectuer, le prix en serait revu à la baisse, déduit du prix de départ. En effet, la durabilité du bâtiment est comprise comme source de variation du prix à la baisse ou à la hausse au regard des marges fixées par le marché pour tel type de bien. "Il y a des travaux d'isolation dans la maison de Madame Martin, alors la tactique à avancer est de pointer les nécessaires travaux de mise en conformité avec les normes éco énergétiques.

L'éco-énergie est alors un espace de valuation du bien à la hausse ou à sa baisse par rapport à son état général défini par les parties et par rapport aux prix du marché pratiqué localement pour le même type de bien.

\section{Quelle place pour la durabilité du bien dans la définition de la valeur du bien ?}


Objet de préoccupation politique à travers les lois et normes qui réglementent le secteur du bâtiment comme indiqué plus haut, la durabilité est aussi une attitude éthique face à la consommation d'énergie et au gaspillage qu'elle recèle. Cette éthique se définit comme l'adoption d'un comportement responsable face à la consommation courante de produits et de services divers. Il s'agit de consommer avec parcimonie, local, en privilégiant la qualité sur la quantité, en choisissant des produits et services dits « responsables » c'est à dire qui intègre les normes de qualité environnementales et sociales, Rumpala (2010).

De ce fait, l'éthique en matière de développement durable se veut être un comportement responsable dans l'optique de préserver et d'économiser les ressources limitées de la planète afin d'assurer aux générations futures des conditions de vie telles que les générations actuelles peuvent les vivre. Ces principes éthiques ont été énoncés et théorisés dans le rapport Brundtland en 1987 qui préconise des comportements à mettre en œuvre au quotidien pour que se réalise un développement durable. Or, nos analyses et enquêtes nous conduisent à observer dans le cadre d'une négociation-vente d'un bien immobilier que la durabilité du bâtiment est un sujet introduit dans le cadre de l'échange pour venir en appoint aux arguments de prix et/ou pour agir sur la sensibilité de l'acquéreur. La négociation/évaluation d'un bien se caractérise par des échanges entre les acteurs de la négociation, ceux-ci évoluent au gré des arguments évoqués sur fond de tactiques pour faire prévaloir ses intérêts. Les modalités de l'échange sont constamment redéfinies au regard des interprétations et des redéfinitions des enjeux et de l'évolution des volontés et intérêts des parties en présence. Stauss (2004). « Nous mobilisons le DPE pour fixer l'attention sur les économies d'énergie donc nous insistons sur la profitabilité que le bâtiment peut receler. En revanche lorsqu'il n'est pas favorable aux économies d'énergie, nous minimisons les coûts d'entrée, les travaux à faire, tout dépend des réactions de l'acheteur, nous vantons les mérites de sa configuration, les agréments liés à l'emplacement, l'exposition au soleil, les services de proximité etc. " "nous n'abordons pas la question éthique dans le cadre d'une vente car nous ne connaissons pas la/les positions des gens par rapport à l'écologie, au développement durable, à la politique, nous ne voulons pas heurter les sensibilités des gens c'est pour ça que les questions de durabilité, d'écologie ne sont pas des points sur lesquelles nous insistons 》 Agent immobilier en transaction.

La durabilité apparait ainsi comme une problématique stratégique dont use avec précaution et prudence les professionnels de la vente de bien immobilier. Le négociateur évoque les termes de prudence, précaution, attention à la sensibilité pour l'éco énergie chez les acheteurs mais rabattus sur la question du prix du bien, revendication explicite de l'acheteur articulée à la négociation sur le prix « il faut faire au préalable les travaux préconisés par les diagnostics». Négociant-acheteur. Cela influe ainsi sur le prix en tant qu'objet de négociation. Aussi, dans le cadre de l'échange, la durabilité est référée au DPE qui traduit les données d'éco-énergie en consommation. Le coût des dépenses à effectuer pour mettre aux normes le bâtiment est discuté entre les parties dans un jeu stratégique. La durabilité du bâtiment est ainsi une variable qui influe sur le prix du bien dans un jeu de valuation structuré autour de ce que (Callon 2017) nomme la recherche de la singularité du bien par les parties. Extraire le bien de l'espace économique et social pour le singulariser, c'est lui attribuer une identité propre et des caractéristiques personnels. Le bien est ainsi valuable en tant que tel, il est objet sur lequel se produit la définition de sa valeur par les parties à la transaction dans un jeu stratégique autour des normes de durabilité construites comme des références éthiques et politiques par les pouvoirs publics.

La durabilité est alors mobilisée et revendiqué par les parties comme espace de référence et d'ajustement du prix aux coûts estimés à effectuer ou à économiser. La durabilité comme 
ensemble de règles que l'on se construit dans un espace socio-culturel, économique et politique donné est ainsi dans ce cadre une variable d'ajustement du prix aux volontés en jeu, et agit comme donnée qui fonde la légitimité de la revendication « du juste prix » pour les négociants. Elle n'est pas invoquée comme croyance sociale désintéressée, donc politique, qui profiterait à l'intérêt commun au détriment de l'intérêt individuel. Elle apparait être une référence qui permet l'étalonnement du prix fondé sur les coûts à économiser ou à supporter.

\section{Cas $N^{\circ} 2$ : Achat d'un immeuble de 4 étages à rénover.}

Le couple Louis achète un immeuble de trois étages à rénover à la périphérie du centre-ville. Leur projet est de placer leurs économies dans l'immobilier. Ils souhaitent le mettre aux normes BBC (bâtiment basse consommation) pour louer les deux T3, les deux T2 et les trois studios. Le vendeur de l'immeuble via son mandataire insiste sur le fait que le coût des travaux sera élevé mais qu'en «tirant» sur les prix des matériaux la facture peut être divisée par deux d'autant plus que Monsieur Louis compte faire l'essentiel des travaux. Il sera aidé par des membres de sa famille ainsi que par des amis. De sensibilité écologiste, Monsieur Louis est sensibilisé aux produits, matériaux et autres équipement peu énergivores. Ils souhaitent équiper son immeuble et ses appartements de tous les équipements de nature à consommer le moins d'énergie possible est ainsi présenter son immeuble et ses appartements comme étant un bâtiment basse consommation dont il espère avoir le label. Or, les matériaux en question sont plus chers et certaines installations nécessitent des travaux plus élaborés, (douche, chaudière, fenêtres triples vitrages, chauffage au sol, adduction d'eau pour alimentation du chauffage urbain, isolation des murs et sols, toiture à changer en partie en équipement velux, système de récupération d'eau... la facture s'avère être élevée et dépasse les prévisions budgétaires du couple Louis. Monsieur Louis indique « il va falloir que je révise mes prétentions car notre budget va exploser si j'équipe mon immeuble de matériaux et équipement BBC et ce malgré les avantages fiscaux. Le coût est élevé. ». le vendeur préconise à Monsieur Louis de s'équiper de matériaux moins chères car il n'entend pas descendre le prix du bien. Il lui recommande des magasins et autres fournisseurs dont les prix sont attractifs en vantant les mérites des produits. "Les matériaux sont tous maintenant de bonne qualité, les fabricants sont attentifs aux économies d'énergies ». Agent immobilier. "Regardez l'emplacement, vous êtes à proximité de tout, vos locataires bénéficient d'un cadre exceptionnel, ils sont à la campagne tout en étant en ville, ce n'est pas mal ça... ça n'a pas de prix, de plus vous êtes en dessous du prix du marché, ce qui vous laisse des marges pour financer les travaux... ».

La négociation sur le prix de l'immeuble va durer plus d'un mois. Le couple Louis va alors mobiliser ses connaissances glanées sur de multiples sites, de multiples " négociants » vont intervenir et faire valoir des arguments techniques pour légitimer soit le prix (côté vendeur) soit sa baisse (côté acheteur). Pour accélérer le processus de vente, l'agent immobilier fait valoir qu'il y a d'autres candidats acheteurs qui semblent eux déterminés et que de ce fait le temps presse. Jouer sur le temps, l'envie, le prix, en référence au marché est une technique bien connue des professionnels de la vente. Le compromis sera finalement signé. Le couple Louis va finalement abandonner l'idée de labeliser le bâtiment BBC du fait du coût d'entrée sans abandonner leur projet de rendre le bâtiment durable. "C'est une question d'économie d'énergie, il faut éviter le gaspillage, à terme on le retrouve sur les factures, c'est un état d'esprit que malheureusement tous n'ont pas. Mais faut bien l'admettre, mettre aux normes $B B C$ un vieux bâtiment ça coûte et la facture est déterminante dans un projet ». Madame Louis.

\subsection{Le coût comme valeur de référence}


La durabilité du bâtiment est représentée et définie à partir du coût dans le cadre d'une transaction. Soit le bâtiment permet d'économiser de l'énergie, cette économie sera traduite en coût donc en gain à espérer, soit elle sera définie en termes de charge pour l'acquéreur. Les calculs autour des coûts apparaissent variables et souvent l'un détermine l'autre dans la négociation. Le coût en termes de charge va être un argument de négociation à la baisse du prix de vente.

En revanche le coût en termes de gains va constituer un argumentaire en faveur du bien. Nous observons que la question éthique n'est pas ou peu mobilisée dans les échanges. Elle vient en appoint, pour donner du crédit à l'argumentaire des parties. De ce fait nous constatons que dans les échanges d'arguments fondés sur la définition du prix du bien une porosité entre les charges et les bénéfices dont peut espérer l'acquéreur. Les gains se définissent par rapport aux charges potentielles et inversement. Le Calcul autour des coûts apparait variable et souvent l'un détermine l'autre dans la négociation. Le coût en termes de charge va être un argument de négociation à la baisse du prix de vente. En revanche le coût en termes de gains va constituer un argumentaire en faveur du bien. Nous observons que la question éthique n'est pas ou peu mobilisée dans les échanges. Elle vient en appoint, pour donner du crédit à l'argumentaire des parties. De ce fait nous observons dans les échanges d'arguments fondés sur la définition du prix du bien une porosité entre les charges et les bénéfices dont peut espérer l'acquéreur. Les gains se définissent par rapport aux charges calculées et inversement. La valeur du bien est constitué ainsi par un certain nombre de paramètres dont on a vu que celui de durabilité est intégré dans le calcul du prix. Il est objet de stratégie au sens où les parties l'instrumentalisent à des fins haussière ou baissière. La valeur attribuée par les parties aux questions de durabilité est lestée par le processus de vente lui-même contraint par la définition du prix en constante négociation. La durabilité est ainsi perçue par les parties comme étant un ensemble de normes techniques et comportementales à mettre en œuvre mais aussi comme une possibilité de « jouer » sur le prix au regard des intérêts des parties en jeux.

\section{Conclusion}

En proposant d'analyser la place de la durabilité d'un bien immobilier dans la négociation/évaluation/et fixation du prix entre un professionnel et un particulier, cet article interroge deux dimensions dans la construction de la valeur d'un bien immobilier, la durabilité et la définition du prix, et pose la question de leur articulation potentielle. Nous avons tenté d'y répondre à travers l'analyse empirique de la construction des négociations/évaluation comme étant une manière de rendre compte des nouvelles formes de négociations, découlant à la fois de la financiarisation et de la durabilité dans le champ de l'immobilier. Elle révèle les capacités dont disposent les acteurs pour accéder à l'information et se construire ainsi une expertise de nature à peser sur la négociation. Les professionnels sont ainsi concurrencés par les profanes dans l'expertise qu'ils apportent lors de la négociation. Les arguments des uns et des autres sont techniques, mais aussi et surtout affectifs, émotionnels et ancrés dans le désir de vendre ou acheter dans le cadre de discours publics sur la durabilité qui se traduisent notamment par des politiques publiques qui tendent à réguler le secteur du bâtiment. Cette régulation se traduit par la production de textes légaux, de normes de construction, de labels, fondées sur une politique et une éthique de la préservation de la planète, Ramade (2005). Cette préservation se matérialise par des incitations et des obligations à se conformer à une pluralité de normes de construction dont l'objectif est d'économiser de l'énergie, de polluer moins, de garantir la santé des habitants par le choix de matériaux appropriés. Passet (1979). Le bâtiment doit être durable au sens où il doit durer dans le temps pour un coût de maintenance moindre. Il est conçu dans l'objectif d'offrir à ses habitants un cadre de vie sains et sécurisé. Ainsi, l'objectif politique et social 
d'allier économie d'énergie, préservation de la santé, de la collectivité, de la planète à travers les gestes quotidien d'attention à la consommation et de construction des bâtiments a redéfinit l'éthique de la consommation des biens et notamment des biens dits immeubles. Burckel \& de Kerangal (2014). La durabilité, dans le cadre d'une négociation relative à la vente d'un bien immobilier apparait être une variable qui joue à la baisse ou à la hausse sur le prix du bien. Ce dernier est objet de redéfinition au regard de l'évolution et de la plausibilité des arguments avancés par les parties. Ces arguments s'inscrivent globalement dans ce qu'il est convenu d'appeler le cadre de la négociation, c'est-à-dire un ensemble de références qui définissent les possibilités de production du prix. Le marché, l'état du bien, l'emplacement, les travaux à réaliser, le lieu de la transaction, la volonté des acteurs.... Ces paramètres donnent du sens et définissent le « cadrage » (Callon 2017) au sein duquel le processus de construction de la valeur du bien par les transactionaires passe par une singularisation de ce dernier, c'est en cela qu'il vaut, car il est singulier dans un espace socio-économique que l'on qualifie par le marché de l'offre et de la demande, espace de comparaison et mesure de la valeur.

Bibliographie

Amarillo H., (2013), Le secteur du bâtiment durable : vers de nouveaux rapports entre acteurs de l'acte de construire ? L'Ires, $n^{\circ} 79$, P.85-109

Bacqué M.H., Biewener C., (2015) L’empowerment, une pratique émancipatrice ? La Découverte. Paris.

Boisnier C., (2015) Les sociétés foncières, entre finance et ville durable, L'Harmattan, Paris.

Burckel D., de Kérangal C. (2014) Sous la dir (Denis Burckel) Management de l'immobilier, Vuibert. Paris.

Callon M. (2017) l'emprise des marchés, La découverte, Paris.

Callon M., Méadel C., Rabeharisoa V., (2000) l'économie des qualités, Politix, Vol. 13, $\mathrm{N}^{\circ}$ 52, P. 211-239

Capron M, Quairel-Lanoizelée F, (2007) La responsabilité sociale d'entreprise, La Découverte. Paris.

Chollet M. (2017) Chez soi, une odyssée de l'espace domestique. La Découverte. SaintAmand-Montrond.

Cochoy Franck \& Dubuisson-Quellier, (2000) les professionnels du marché : vers une sociologie du travail marchand. Sociologie du travail, vol $42, n^{\circ} 3$ p.p. $357-520$

Coloos B. (1997) dir Comprendre les marchés du logement, L’Harmatan. Paris. 
Cusin F., (2004) in Cochoy (dir) Attirer, sélectionner, fidéliser : le double marché du crédit aux particuliers, PUM. Toulouse.

Dubar C, Tripier P, Boussard V, (2015) Sociologie des professions, Armand Colin, Paris.

Ducroux, A.M. (2002). Les nouveaux utopistes du développement durable. Autrement, Paris.

Dubuisson-Quellier (2018) La consommation engagée, Presses de Sciences Po, Paris.

.Dupont C. (1994) la négociation : conduite, théorie, applications, Dalloz, Paris.

Favereau O., (1989), Marchés internes, marchés externes, Revue économique, Vol. 40, $\mathrm{n}^{\circ} 2$ p.p. 276-328.

Fligstein N., (2001) Le mythe du marché, Actes de la recherche en sciences sociales, $\mathrm{n}^{\circ} 139$, sept., P. 3-12

Flipo F. (2011) Le développement durable. Bréal, Monts.

Granovetter, M (2000). Le marché autrement, Les réseaux dans l'économie, Desclée de Brouwer, Paris.

Hlady-Rispal M. (2000), « Une stratégie de recherche en gestion : l'étude de cas », Revue française de gestion, janvier-février, p. 61-70.

Karpik L (2007), l'économie des singularités, bibliothèques des sciences humaines, Gallimard, Paris.

Larceneux F., Parent H., (2010) Marketing de l'Immobilier. Dunod. Paris.

Legendre R. (1993) Dictionnaire actuel de 'l'éducation, Guérin, Eska.. Paris.

Nappi-Choulet I., (2009) Les mutations de l'immobilier, de la finance au développement durable, Autrement. Paris.

Passet R., (1979), L'économique et le vivant, Payot, Paris.

Paul G. (2011) Le Grenelle de l'environnement, En poche, Gualino, Lextenso éditions. Paris.

Primault L. (2015) Bâtir une politique durable du logement. Les pratiques. Le passager clandestin. Neuvy-en-Champagne.

Ramade F., (2005), Ecologie Générale, Dunod, Paris.

Rivaton R., Pavanello V., (2017) L’immobilier demain, Dunod. Paris.

Rumpala Y. (2010) Développement durable, ou le gouvernement du changement local. Le Bord de L'eau. Paris. 
Steiner P. (1998) La nouvelle sociologie économique, l'analyse structurale et la théorie économique, Cahiers d'Economie Politique, 1998, vol 33, pp. 107-136.

Stimec A. (2011) la négociation. Dunod. Paris.

Strauss A. (2004) La trame de la négociation. Sociologie qualitative et interactionnisme. Logiques Sociales, L'Harmattan. Paris.

Trepos J.Y, (1996) La sociologie de l'expertise, Coll Que-sais-je ? Paris.

Trigilia C, (2002) Sociologie économique, Coll U Armand Collin, Paris.

Vatin F., (dir) (2009) Evaluer et valoriser. Une sociologie économique de la mesure. PUM. Toulouse.

Weber M. (1995), Economie et société, Pocket, Paris.

Yché A. (2011) Logement, habitat \& cohésion sociale, au-delà de la crise, quelle société voulons-nous demain? Mollat. Castuera. Espagne.

Zelizer V. A., (2001) transactions intimes, Genèses, vol 42, mars, P. 121-144 